\title{
On approximation properties of spline based $F$-transform with respect to fuzzy $m$-partition
}

\author{
Irina Kodorane ${ }^{1}$ Svetlana Asmuss $^{1,2}$ \\ ${ }^{1}$ Department of Mathematics, University of Latvia \\ ${ }^{2}$ Institute of Mathematics and Computer Science of University of Latvia
}

\begin{abstract}
In this paper we develop spline based $F$-transforms by considering fuzzy partitions given by polynomial splines. We investigate approximation properties of spline based $F^{(m)}$-transforms and prove that using generalized fuzzy $m$-partition, which consists of basic functions of bandwidth $m$, allows us to approximate not only original function, but also its derivatives.
\end{abstract}

Keywords: Fuzzy partition, Spline, Generalized fuzzy partition, Fuzzy transform, Approximation property, Approximation of derivatives

\section{Introduction}

The direct and inverse fuzzy transforms ( $F$ transforms) have been introduced by I. Perfilieva [6]. Theory and applications of $F$-transforms in image processing, data analysis and time series analysis have been actively developed during last years (see e.g. [3], [4], [5], [7], [9], [11]). The technique of $F$ transforms has been generalized in many directions: discrete $F$-transforms, higher order $F$-transforms [8],[10], $F$-transforms with respect to a generalized fuzzy $m$-partition $\left(F^{(m)}\right.$-transforms) [12]. For each type of fuzzy transforms approximation properties have been investigated.

Spline based $F$-transforms have been considered by B. Bede and J.R. Rudas [1] in the case when a fuzzy partition is given by means of B-splines. In the framework of the classical approximation theory they obtained new error estimations for such type of fuzzy transforms. The aim of our paper is to suggest an alternative approach and to introduce a new type of spline based fuzzy partitions. Our main attention is paid to generalized fuzzy $\mathrm{m}$ partitions of bandwidth $m$ defined by using polynomial splines of degree $m$. We prove that polynomial spline based inverse $F^{(m)}$-transform with respect to generalized fuzzy $m$-partitions approximates not only the original function $f$ itself, but also its derivatives $f^{\prime}, f^{\prime \prime}, \ldots, f^{m-1}$.

Let us note that I. Perfilieva and V. Kreinovich in their paper [10] proved good approximation properties considering approximation of derivatives in the case of higher order fuzzy transforms.

\section{Preliminary}

We briefly recall the basic definitions of the Ftransform and related notions introduced by I. Perfilieva [6].

Definition 1. Let $x_{1}<x_{2}<\ldots<x_{n}$ be fixed nodes within $[a, b]$, such that $x_{1}=a, x_{n}=b$ and $n \geq 2$. We say that fuzzy sets $A_{1}, A_{2}, \ldots, A_{n}$, identified with their membership functions (basic functions) defined on $[a, b]$, form fuzzy partition of $[a, b]$ if they fulfill the following conditions for $k=1,2, \ldots, n$ :

1) $A_{k}:[a, b] \rightarrow[0,1], A_{k}\left(x_{k}\right)=1$;

2) $A_{k}(x)=0$, if $x \notin\left(x_{k-1}, x_{k+1}\right)$, where for the uniformity of denotion, we put $x_{0}=a$ and $x_{n+1}=b$;

3) $A_{k}$ is continuous;

4) $A_{k}$, where $k=2, \ldots, n$, strictly increases on $\left[x_{k-1}, x_{k}\right]$ and $A_{k}$, where $k=1, \ldots, n-1$, strictly decreases on $\left[x_{k}, x_{k+1}\right]$;

5) for all $x \in[a, b]$

$$
\sum_{k=1}^{n} A_{k}(x)=1
$$

In the case of equidistant nodes $x_{1}, x_{2}, \ldots, x_{n}$ two additional properties of basic functions $A_{k}$ for $k=2, \ldots, n$ fulfil:

6) $A_{k}\left(x_{k}-x\right)=A_{k}\left(x_{k}+x\right)$ for all $x \in[0, h]$;

7) $A_{k}(x)=A_{k-1}(x-h)$ for all $x \in\left[x_{k}, x_{k+1}\right]$, and $A_{k+1}(x)=A_{k}(x-h)$ for all $x \in\left[x_{k-1}, x_{k}\right]$;

where $h=(b-a) /(n-1)$.

Definition 2. Let $A_{1}, A_{2}, \ldots, A_{n}$ be basic functions which form a fuzzy partition of $[a, b], n \geq 2$, and $f$ be any function from $C([a, b])$. We say that the $n$ tuple $F[f]=\left(F_{1}, F_{2}, \ldots, F_{n}\right)$ given by

$$
F_{k}=\frac{\int_{a}^{b} f(x) A_{k}(x) d x}{\int_{a}^{b} A_{k}(x) d x}, k=1,2, \ldots, n,
$$

is the F-transform of $f$ with respect to $A_{1}, A_{2}, \ldots, A_{n}$.

If fuzzy partition $A_{1}, A_{2}, \ldots, A_{n}$ of $[a, b]$ is uniform then expressions for components of the $F$ - 
transforms may be simplified as follows

$$
\begin{aligned}
& F_{1}=\frac{2}{h} \int_{x_{1}}^{x_{2}} f(x) A_{1}(x) d x ; \\
& F_{k}=\frac{1}{h} \int_{x_{k-1}}^{x_{k+1}} f(x) A_{k}(x) d x, k=2, \ldots, n-1 ; \\
& F_{n}=\frac{2}{h} \int_{x_{n-1}}^{x_{n}} f(x) A_{n}(x) d x .
\end{aligned}
$$

Definition 3. Let $F[f]=\left(F_{1}, F_{2}, \ldots, F_{n}\right)$ be the $F$-transform of a continuous function $f \in C([a, b])$ with respect to a fuzzy partition $A_{1}, A_{2}, \ldots, A_{n}$ of $[a, b], n \geq 2$. Then the function

$$
f_{F, n}(x)=\sum_{k=1}^{n} F_{k} A_{k}(x), x \in[a, b],
$$

is called the inverse $F$-transform of $f$ with respect to $A_{1}, A_{2}, \ldots, A_{n}$.

\section{Spline based F-transforms}

Our paper deals with spline based fuzzy transforms using fuzzy partitions described by polynomial splines.

We consider the case of equidistant nodes

$$
a=x_{1}<x_{2}<\ldots<x_{n}=b, n \geq 2,
$$

and use the notation

$$
\Delta_{n}(a, b)=\left\{x_{k}=a+h(k-1) \mid k=1,2, \ldots, n\right\},
$$

where $h=(b-a) /(n-1)$. To introduce basic functions by using polynomial splines of degree $m \geq 1$ we consider additional equidistant nodes by dividing each interval $\left[x_{k-1}, x_{k}\right], k=2, \ldots, n$, into $m$ subintervals. Let us denote obtained partition

$\Delta_{N}(a, b)=\left\{t_{1}, t_{2}, \ldots, t_{N} \mid t_{i}=a+(i-1) /(N-1)\right\}$, where $N=m(n-1)+1$ and for all $k=1, \ldots, n-1$ $x_{k}=t_{m(k-1)+1}<t_{m(k-1)+2}<\ldots<t_{m k+1}=x_{k+1}$, and describe the space $S_{m, 1}\left(\Delta_{N}(a, b)\right)$ of polynomial splines of degree $m$ and defect 1 with respect to $\Delta_{N}(a, b)$ (see e.g. [2],[13] ).

Definition 4. Let $\Delta_{N}(a, b)=\left\{t_{1}, t_{2}, \ldots, t_{N}\right\}$ be $a$ partition of an interval $[a, b], N \geq 2$. We say that a function $S:[a, b] \rightarrow \mathbb{R}$ is a spline of degree $m \geq$ 1 and defect 1 with respect to $\Delta_{N}(a, b)$ (i.e. $S \in$ $\left.S_{m, 1}\left(\Delta_{N}(a, b)\right)\right)$ if it fulfils the following conditions:

1) $S$ is a polynomial of degree not greater than $m$ on each interval $\left[t_{i-1}, t_{i}\right], i=2, \ldots, N$;

2) $S$ is $m-1$ times continuously differentiable on interval $[a, b]$, i.e. $S \in C^{m-1}([a, b])$.

It is known that $S \in S_{m, 1}\left(\Delta_{N}(a, b)\right)$ if and only if there exist coefficients $\alpha_{0}, \alpha_{1}, \ldots, \alpha_{m}$, $\beta_{2}, \ldots, \beta_{N-1} \in \mathbb{R}$ such that

$$
S(x)=\sum_{j=0}^{m} \alpha_{j}(x-a)^{j}+\sum_{i=2}^{N-1} \beta_{i}\left(x-t_{i}\right)_{+}^{m}, x \in[a, b],
$$

where

$$
\left(x-t_{i}\right)_{+}^{m}=\left\{\begin{array}{l}
\left(x-t_{i}\right)^{m}, \text { if } x \geq t_{i}, \\
0, \text { if } x<t_{i} .
\end{array}\right.
$$

Proposition 5. Let $\Delta_{m+1}(0, h)=\left\{\tau_{1}, \ldots, \tau_{m+1}\right\}$ be the uniform partition of interval $[0, h]$ for a given integer $m \geq 1$ and positive $h \in \mathbb{R}$. There exists the unique spline $S^{m} \in S_{m, 1}\left(\Delta_{m+1}(0, h)\right)$ such that:
1) $\left(S^{m}\right)^{(r)}(0)=0$ for $r=0,1, \ldots, m-1$;
2) $\left(S^{m}\right)(h)=1$;
3) $\left(S^{m}\right)^{(r)}(h)=0$ for $r=1,2, \ldots, m-1$;
4) $S^{m}$ strictly increases on $[0, h]$;
5) $S^{m}(t)+S^{m}(h-t)=1$ for all $t \in[0, h]$.

Proof. To find spline $S^{m} \in S_{m, 1}\left(\Delta_{m+1}(0, h)\right)$, which fulfils conditions 1)-5), we use general representation formula

$$
S^{m}(t)=\sum_{i=1}^{m} \beta_{i}\left(t-\tau_{i}\right)_{+}^{m}, t \in[0, h],
$$

taking into account that $\alpha_{j}=0$ for $j=0,1, \ldots, m-1$ and $\alpha_{m}=\beta_{1}$.

Condition 3) gives us the system of linear equations, whose determinant

$$
\left|\begin{array}{cccc}
(m-1)^{m} & (m-2)^{m} & \ldots & 1 \\
(m-1)^{m-1} & (m-2)^{m-1} & \ldots & 1 \\
\ldots & \ldots & \ldots & \ldots \\
(m-1) & (m-2) & \ldots & 1
\end{array}\right|
$$

does not equal to 0 . This means that there exists the unique spline $S^{m} \in S_{m, 1}\left(\Delta_{m+1}(0, h)\right)$, which fulfils conditions 1$)-3$ ). To prove the monotonicity of this spline (condition 4) ) we find a new representation for $S^{m}$ :

$$
S^{m}(t)=\int_{0}^{t} B^{m-1}(\tau) d \tau, t \in[0, h],
$$

where $B^{m-1}$ is the $B$-spline from the space $S_{m-1,1}\left(\Delta_{m+1}(0, h)\right)$, which support equals to $[0, h]$ and which is normed by the equality

$$
\int_{0}^{h} B^{m-1}(\tau) d \tau=1
$$

Now the inequality

$$
\left(S^{m}\right)^{\prime}(t)=B^{m-1}(t)>0, t \in(0, h)
$$

implies the monotonicity of $S^{m}$.

To complete the proof it remains to verify condition 5). Taking into account that the equality $S^{m}(t)+S^{m}(h-t)=1$ is true for $t=0$ and $t=h$, we need to prove that the sum $S^{m}(t)+S^{m}(h-t)$ is constant on $[0, h]$. It follows from the fact that

$\left(S^{m}\right)^{\prime}(t)-\left(S^{m}\right)^{\prime}(h-t)=B^{m-1}(t)-B^{m-1}(h-t)=0$

for all $t \in[0, h]$. Now by using spline $S^{m} \in$ $S_{m, 1}\left(\Delta_{m+1}(0, h)\right)$ from Proposition 5 we introduce spline based fuzzy partition

$$
S_{1}^{m}, S_{2}^{m}, \ldots, S_{n}^{m} \in S_{m, 1}\left(\Delta_{N}(a, b)\right),
$$


$m \geq 1$, applying the formula:

$$
\begin{gathered}
S_{k}^{m}(x)=\left\{\begin{array}{l}
S^{m}\left(x-x_{k-1}\right), x \in\left[x_{k-1}, x_{k}\right] \\
S^{m}\left(x_{k+1}-x\right), x \in\left[x_{k}, x_{k+1}\right] \\
0, \quad \text { otherwise },
\end{array}\right. \\
k=1,2, \ldots, n .
\end{gathered}
$$

For the uniformity of denotation we put $x_{0}=a-h$, $x_{n+1}=b+h$ and define $S_{1}^{m}$ for $x \in\left[x_{0}, x_{1}\right)$ and $S_{n}^{m}$ for $x \in\left(x_{n}, x_{n+1}\right]$.

The first degree splines $(m=1)$ describe triangular shaped basic functions. Next we give the second degree (quadratic) and the third degree (cubic) splines obtained as uniform fuzzy partition basic functions:

$S_{k}^{2}(x)=\left\{\begin{array}{l}\frac{2\left(x-x_{k-1}\right)^{2}}{h^{2}}, x \in\left[x_{k-1}, x_{k-1}+\frac{h}{2}\right], \\ 1-\frac{2\left(x-x_{k}\right)^{2}}{h^{2}}, x \in\left[x_{k-1}+\frac{h}{2}, x_{k}+\frac{h}{2}\right], \\ \frac{2\left(x-x_{k+1}\right)^{2}}{h^{2}}, x \in\left[x_{k}+\frac{h}{2}, x_{k+1}\right], \\ 0, \text { otherwise, }\end{array}\right.$

$S_{k}^{3}(x)=\left\{\begin{array}{l}\frac{9\left(x-x_{k-1}\right)^{3}}{2 h^{3}}, x \in\left[x_{k-1}, x_{k-1}+\frac{h}{3}\right], \\ \frac{1}{2}+\frac{9\left(x-x_{k-1}-\frac{h}{2}\right)}{4 h}-\frac{9\left(x-x_{k-1}-\frac{h}{2}\right)^{3}}{h^{3}}, \\ 1+\frac{9\left(x-x_{k}\right)^{3}}{2 h^{3}}, x \in\left[x_{k-1}+\frac{2 h}{3}, x_{k}\right], \\ 1-\frac{9\left(x-x_{k}\right)^{3}}{2 h^{3}}, x \in\left[x_{k}, x_{k}+\frac{h}{3}\right], \\ \frac{1}{2}-\frac{9\left(x-x_{k}-\frac{h}{2}\right)}{4 h}+\frac{9\left(x-x_{k}-\frac{h}{2}\right)^{3}}{h^{3}}, \\ -\frac{9\left(x-x_{k+1}\right)^{3}}{2 h^{3}}, x \in\left[x_{k}+\frac{h}{3}, x_{k}+\frac{2 h}{3}\right], \\ 0, \quad \text { otherwise, }\end{array}\right.$

$$
k=1,2, \ldots, n \text {. }
$$

Fig.1 shows an example of spline based fuzzy partition.

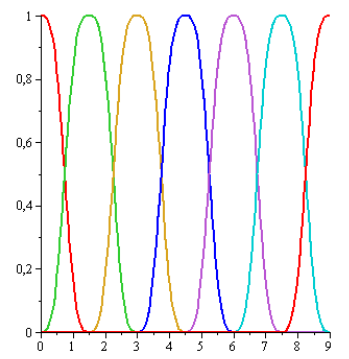

Figure 1: Cubic spline based uniform fuzzy partition of $[0,9], \mathrm{n}=7$.

Let the uniform fuzzy partition of $[a, b]$ be given by splines $S_{1}^{m}, S_{2}^{m}, \ldots, S_{n}^{m}, n \geq 2, m \geq 1$. We denote by $F^{m}[f]=\left(F_{1}^{m}, F_{2}^{m}, \ldots, F_{n}^{m}\right)$ the $\bar{F}$-transform of a continuous function $f$ from $C([a, b])$ with respect to $S_{1}^{m}, S_{2}^{m}, \ldots, S_{n}^{m}$ :

$$
F_{k}^{m}=\frac{\int_{a}^{b} f(x) S_{k}^{m}(x) d x}{\int_{a}^{b} S_{k}^{m}(x) d x}, k=1,2, \ldots, n,
$$

and consider the corresponding inverse $F$-transform

$$
f_{F, n}^{m}(x)=\sum_{k=1}^{n} F_{k}^{m} S_{k}^{m}(x), x \in[a, b] .
$$

An example of the inverse $F$-transform with respect to cubic spline based universe fuzzy partition is given by Fig. 2 .

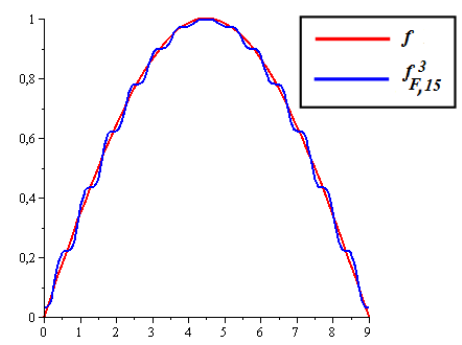

Figure 2: Test function $f(x)=\sin (x \pi / 9)$ and its inverse $F$-transform with respect to cubic spline based uniform fuzzy partition, $\mathrm{n}=15$.

In numerical experiments it became clear that by using generalized fuzzy $m$-partitions we can significantly reduce the oscillation of the inverse transformation. One can easy compare Fig. 2 with Fig. 4 from the next Section.

\section{Spline based $F^{(m)}$-transforms with respect to generalized fuzzy $m$-partition}

Each basic function $A_{k}$ of a fuzzy partition $A_{1}$, $A_{2}, \ldots, A_{n}$ over $\Delta_{n}(a, b)$ has been considered to be zero outside the union of two adjacent subintervals $\left[x_{k-1}, x_{k}\right] \cup\left[x_{k}, x_{k+1}\right]$. L. Stefanini [12] generalized the concept of a fuzzy partition by taking basic functions which support covers more than two consecutive subintervals. For generalized basic function $A_{k}^{(m)}, m \geq 1$, he considered $2 m+1$ consecutive points (and consequently $2 m$ subintervals) of $\Delta_{n}(a, b): x_{k-m}, \ldots, x_{k}, \ldots, x_{k+m}$. For the correct notation we extend the partition $\Delta_{n}(a, b)$ by taking additional points:

$x_{-2 m+2}<\ldots<x_{0}<a, b<x_{n+1}<\ldots<x_{n+2 m-1}$.

In the case of uniform partition with $h=\frac{b-a}{n-1}$ the additional points are:

$$
\begin{gathered}
x_{i}=a+(i-1) h, \\
i=-2 m+2,-2 m+3, \ldots, 0, n+1, n+2, \ldots, n+2 m-1 .
\end{gathered}
$$

Let us denote this extension by $\Delta_{n, m}(a, b)$. For

$$
a_{m}=a-(2 m-1) h \text { and } b_{m}=b+(2 m-1) h
$$

we have $\Delta_{n, m}(a, b)=\Delta_{n+4 m-2}\left(a_{m}, b_{m}\right)$. 
Definition 6. Let $\Delta_{n}(a, b)=\left\{x_{1}, \ldots, x_{n}\right\}$ be the uniform partition of $[a, b], n \geq 2$, and let

$$
\Delta_{n, m}(a, b)=\left\{x_{-2 m+2}, x_{-2 m+3}, \ldots, x_{n+2 m-1}\right\}
$$

be its uniform extension, $m \geq 1$. We say that fuzzy sets $A_{-m+2}^{(m)}, A_{-m+3}^{(m)}, \ldots, A_{n+m-1}^{(m)}$, identified with their membership functions (basic functions of bandwidth $m$ ) defined on $\left[a_{m}, b_{m}\right]$, where $a_{m}=$ $a-(2 m-1) h$ and $b_{m}=b+(2 m-1) h, h=$ $(b-a) /(n-1)$, form uniform generalized fuzzy $m$ partition of $[a, b]$ if they fulfil the following conditions for $k=m-2, m-1, \ldots, n+m-1$ :

1) $A_{k}^{(m)}:\left[a_{m}, b_{m}\right] \longrightarrow[0,1], A_{k}^{(m)}\left(x_{k}\right)=1$;

2) $A_{k}^{(m)}(x)=0$ for $x \notin\left[x_{k-m}, x_{k+m}\right]$;

3) $A_{k}^{(m)}$ is continuous;

4) $A_{k}^{(m)}$ is decreasing on $\left[x_{k}, x_{k+m}\right]$;

5) $A_{k}^{(m)}$ is increasing on $\left[x_{k-m}, x_{k}\right]$;

6) $A_{k}^{(m)}\left(x_{k}-x\right)=A_{k}^{(m)}\left(x_{k}+x\right)$ for all $x \in[0, m h]$

7) $A_{k}^{(m)}(x)=A_{k-1}^{(m)}(x-h)$ for all $x \in\left[x_{k-m}, x_{k+m}\right]$, if $k=-m+3, \ldots, n+m-1 ;$

8) for all $x \in[a, b]$

$$
\sum_{k=-m+2}^{n+m-1} A_{k}^{(m)}(x)=m
$$

It is known that in the case of uniform fuzzy $m$ partition for each $k=-m+2, \ldots, n+m-1$

$$
\int_{x_{k-m}}^{x_{k+m}} A_{k}^{(m)}(x) d x=m h .
$$

We introduce basic functions of bandwidth $m$ by using polynomial splines from $S_{m, 1}\left(\Delta_{n+4 m-2}\left(a_{m}, b_{m}\right)\right)$. Transformation from spline based fuzzy partition to spline based fuzzy $m$-partition can be realized according to the following scheme. By using spline $S^{m} \in S_{m, 1}\left(\Delta_{m+1}(0, h)\right)$ from Proposition 5 we obtain spline $S^{(m)} \in S_{m, 1}\left(\Delta_{m+1}(0, m h)\right)$ :

$$
S^{(m)}(t)=S^{m}\left(\frac{t}{m}\right), t \in[0, m h],
$$

and apply it to define

$$
S_{k}^{(m)} \in S_{m, 1}\left(\Delta_{n+4 m-2}\left(a_{m}, b_{m}\right)\right)
$$

as follows:

$$
\begin{gathered}
S_{k}^{(m)}(x)= \begin{cases}S^{(m)}\left(x-x_{k-m}\right), & x \in\left[x_{k-m}, x_{k}\right], \\
S^{(m)}\left(x_{k+m}-x\right), & x \in\left[x_{k}, x_{k+m}\right], \\
0, & \text { otherwise },\end{cases} \\
k=-m+2,-m+3, \ldots, n+m-1 .
\end{gathered}
$$

We give the formulas obtained for spline based generalized fuzzy $m$-partition, when $m=2,3$ :

$$
S_{k}^{(2)}(x)=\left\{\begin{array}{l}
\frac{1}{2} \frac{\left(x-x_{k-2}\right)^{2}}{h^{2}}, x \in\left[x_{k-2}, x_{k-1}\right], \\
1-\frac{1}{2} \frac{\left(x-x_{k}\right)^{2}}{h^{2}}, x \in\left[x_{k-1}, x_{k+1}\right], \\
\frac{1}{2} \frac{\left(x-x_{k+2}\right)^{2}}{h^{2}}, x \in\left[x_{k+1}, x_{k+2}\right], \\
0, \text { otherwise, }
\end{array}\right.
$$

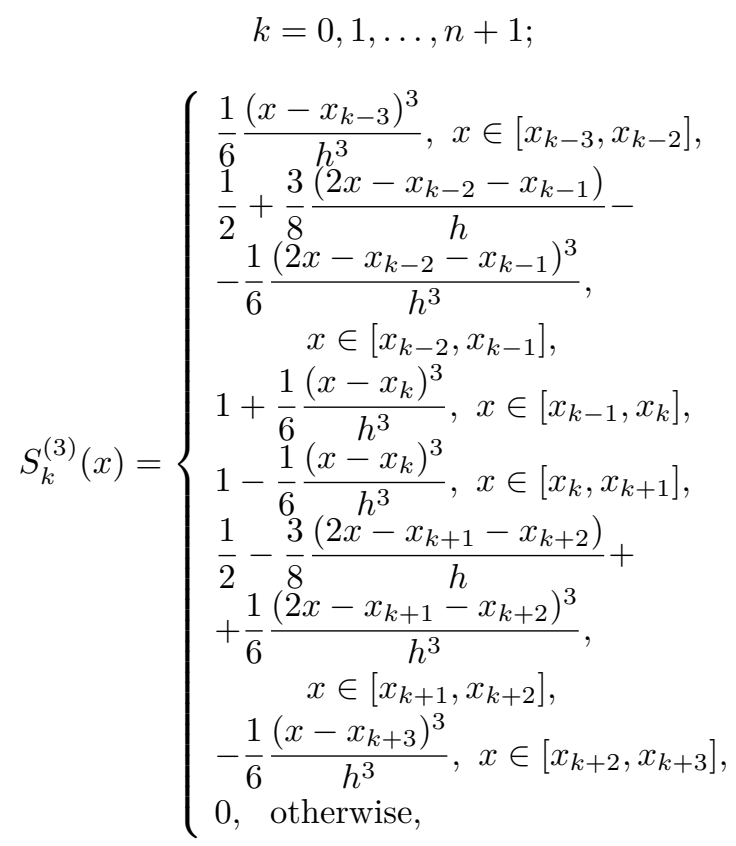

$$
k=-1,0, \ldots, n+2 .
$$

Fig.3 shows an example of spline based generalized fuzzy $m$-partition.

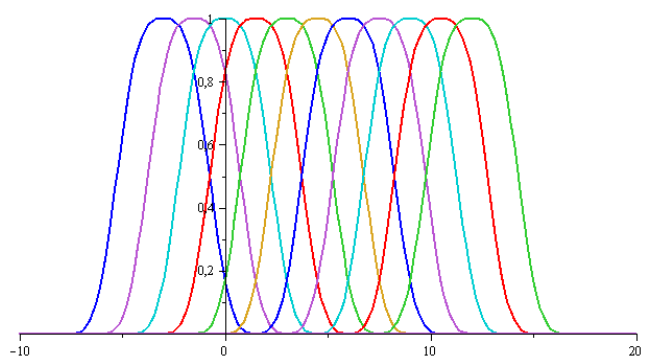

Figure 3: Cubic spline based uniform fuzzy 3partition of $[0,9], \mathrm{n}=7$.

Let the uniform fuzzy $m$-partition of $[a, b]$ be given by splines $S_{-m+2}^{(m)}, S_{-m+3}^{(m)}, \ldots, S_{n+m-1}^{(m)}$, $n \geq 2$ and $m \geq 1$. For a continuous function $f$ from $C\left(\left[a_{m}, b_{m}\right]\right)$ we consider the direct $F^{(m)}$-transform based on $S_{-m+2}^{(m)}, S_{-m+3}^{(m)}, \ldots, S_{n+m-1}^{(m)}$, which is defined by the vector $F^{(m)}[f]=\left(F_{-m+2}^{(m)}, F_{2}^{(m)}, \ldots, F_{n+m-1}^{(m)}\right)$ with components

$$
F_{k}^{(m)}=\frac{1}{m h} \int_{x_{k-m}}^{x_{k+m}} f(x) S_{k}^{(m)}(x) d x,
$$


$k=-m+2, \ldots, n+m-1$, and the corresponding inverse $F^{(m)}-$ transform function

$$
f_{F, n}^{(m)}(x)=\frac{1}{m} \sum_{k=-m+2}^{n+m-1} F_{k}^{(m)} S_{k}^{(m)}(x), x \in[a, b] .
$$

An example of the inverse $F^{(m)}$-transform with

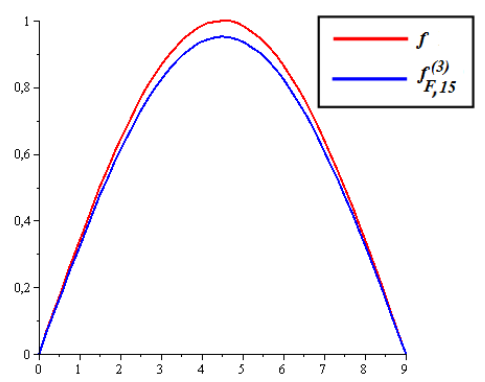

Figure 4: Test function $f(x)=\sin (x \pi / 9)$ and its inverse $F^{(3)}$-transform with respect to cubic spline based uniform fuzzy 3 -partition, $\mathrm{n}=15$.

respect to spline based universe fuzzy partition is given by Fig. 4. Numerical experiments show, that $F^{(m)}$-transforms can be effectively used to approximate derivatives of an original function (see Fig. 5 and Fig. 6).

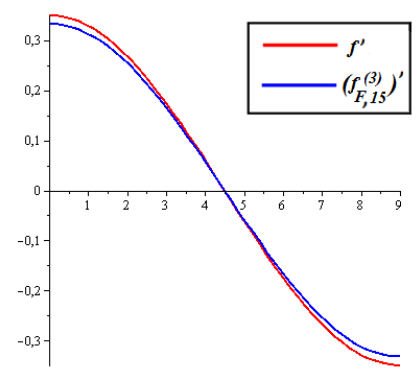

Figure 5: The first derivative of test function $f(x)=\sin (x \pi / 9)$ and of its inverse $F^{(3)}$-transform with respect to cubic spline based uniform fuzzy 3 partition, $\mathrm{n}=15$.

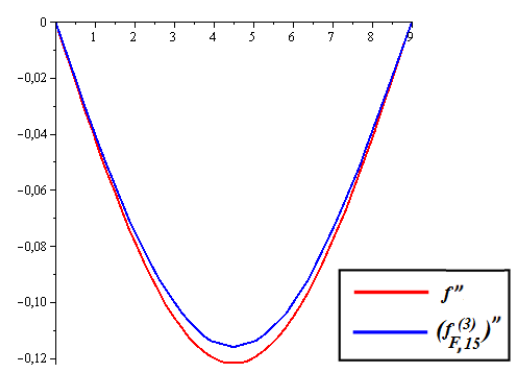

Figure 6: The second derivative of test function $f(x)=\sin (x \pi / 9)$ and of its inverse $F^{(3)}$-transform with respect to cubic spline based uniform fuzzy 3partition, $\mathrm{n}=15$.

\section{Approximation properties of $F^{(m)}$-transforms}

In order to obtain the error estimation for the inverse $F^{(m)}$-transform we need the definition of the modulus of continuity.

Definition 7. Let $f$ be a function from $C([a, b])$ and $\delta$ be a positive number. Then $\omega(\delta, f)$ given by

$\omega(\delta, f)=\max \{|f(x)-f(y)||x, y \in[a, b]| x-y \mid, \leq \delta\}$ is called the modulus of continuity of $f$ on $[a, b] a c$ cording to $\delta$.

Theorem 8. Let $f$ be a function from $C\left(\left[a_{m}, b_{m}\right]\right)$ and $f_{F, n}^{(m)}$ be its inverse $F^{(m)}$-transform with respect to the spline based uniform generalized fuzzy $m$-partition $S_{-m+2}^{(m)}, S_{-m+3}^{(m)}, \ldots, S_{n+m-1}^{(m)}$ of $[a, b]$, where

$$
\begin{gathered}
a_{m}=a-(2 m-1) h, b_{m}=b+(2 m-1) h, \\
h=(b-a) /(n-1), m \geq 1, n \geq 2 .
\end{gathered}
$$

Then for all $x \in[a, b]$ holds

$$
\left|f(x)-f_{F, n}^{(m)}(x)\right| \leq \omega(2 m h, f),
$$

where $\omega(2 m h, f)$ is the modulus of continuity of $f$ on $\left[a_{m}, b_{m}\right]$ according to $2 m h$.

Proof. We start with the proof of the inequality

$$
\left|f(x)-F_{k}^{(m)}\right| \leq \omega(2 m h, f)
$$

for each $k=-m+2, \ldots, n+m-1$ and for each $x \in\left[x_{k-m}, x_{k+m}\right]$.

If $t \in\left[x_{k-m}, x_{k+m}\right]$, then

$$
\begin{gathered}
\left|f(x)-F_{k}^{(m)}\right|= \\
=\left|\frac{1}{m h} \int_{x_{k-m}}^{x_{k+m}}(f(x)-f(t)) S_{k}^{(m)}(t) d t\right| \leq \\
\leq \frac{\omega(2 m h, f)}{m h} \int_{x_{k-m}}^{x_{k+m}} S_{k}^{(m)}(t) d t=\omega(2 m h, f) .
\end{gathered}
$$

Now lets assume that $x \in\left[x_{k}, x_{k+1}\right]$ for some $k=$ $1, \ldots, n-1$, and rewrite $f(x)-f_{F, n}^{(m)}(x)$ by using the equality

$$
\sum_{i=k-m+1}^{k+m} S_{i}^{(m)}(x)=m
$$

as follows

$$
\begin{gathered}
f(x)-f_{F, n}^{(m)}(x)= \\
=\frac{1}{m} \sum_{i=k-m+1}^{k+m} S_{i}^{(m)}(x)\left(f(x)-F_{i}^{(m)}\right) .
\end{gathered}
$$

Therefore

$$
\begin{gathered}
\left|f(x)-f_{F, n}^{(m)}(x)\right| \leq \\
\leq \frac{\omega(2 m h, f)}{m} \sum_{i=k-m+1}^{k+m} S_{i}^{(m)}(t)=\omega(2 m h, f) .
\end{gathered}
$$


Remark 9. Let us note that the proof of the previous theorem does not use the special form of basic functions. It means that this result is true for any uniform generalized fuzzy m-partition.

The following theorems will show derivative approximation error bounds in the case of quadratic and cubic spline based fuzzy $m$-partition, when $m=2$ and $m=3$.

Theorem 10. Let $f$ be a function from $C^{1}\left(\left[a_{2}, b_{2}\right]\right)$ (i.e. continuously differentiable on interval $\left[a_{2}, b_{2}\right]$ ) and $f_{F, n}^{(2)}$ be its inverse $F^{(2)}$-transform with respect to the second degree spline based uniform generalized fuzzy 2-partition of $[a, b]$, where

$a=a_{2}+3 h, b=b_{2}-3 h, h=(b-a) /(n-1), n \geq 2$.

Then for all $x \in[a, b]$ holds

$$
\left|f^{\prime}(x)-\left(f_{F, n}^{(2)}\right)^{\prime}(x)\right| \leq \omega\left(4 h, f^{\prime}\right)
$$

where $\omega\left(4 h, f^{\prime}\right)$ is the modulus of continuity of $f^{\prime}$ on $\left[a_{2}, b_{2}\right]$ according to $4 h$.

Proof. By differentiating the formula

$$
f_{F, n}^{(2)}(x)=\frac{1}{2} \sum_{k=0}^{n+1} F_{k}^{(2)} S_{k}^{(2)}(x)
$$

where

$$
F_{k}^{(2)}=\frac{1}{2 h} \int_{x_{k-2}}^{x_{k-2}} f(x) S_{k}^{(2)}(x) d x
$$

and

$$
\int_{x_{k-2}}^{x_{k-2}} S_{k}^{(2)}(x) d x=2 h
$$

we obtain that the derivative

$$
\left(f_{F, n}^{(2)}\right)^{\prime}(x)=\frac{1}{2} \sum_{k=0}^{n+1} F_{k}^{(2)}\left(S_{k}^{(2)}\right)^{\prime}(x)
$$

is the first degree spline with values

$$
\left(f_{F, n}^{(2)}\right)^{\prime}\left(x_{k}\right)=\frac{1}{2} \sum_{i=k-2}^{k+2} F_{i}^{(2)}\left(S_{i}^{(2)}\right)^{\prime}\left(x_{k}\right) .
$$

By inserting the values

$$
\begin{gathered}
\left(S_{k-2}^{(2)}\right)^{\prime}\left(x_{k}\right)=\left(S_{k}^{(2)}\right)^{\prime}\left(x_{k}\right)=\left(S_{k+2}^{(2)}\right)^{\prime}\left(x_{k}\right)=0, \\
\left(S_{k-1}^{(2)}\right)^{\prime}\left(x_{k}\right)=-\frac{1}{h} \text { and }\left(S_{k+1}^{(2)}\right)^{\prime}\left(x_{k}\right)=\frac{1}{h},
\end{gathered}
$$

we get

$$
\left(f_{F, n}^{(2)}\right)^{\prime}\left(x_{k}\right)=\frac{F_{k+1}^{(2)}-F_{k-1}^{(2)}}{2 h}
$$

and express $F_{k+1}^{(2)}$ and $F_{k-1}^{(2)}$ by using $S_{k}^{(2)}$ :

$$
\text { 1) } \begin{aligned}
& F_{k+1}^{(2)}=\frac{1}{2 h} \int_{x_{k-1}}^{x_{k+3}} f(x) S_{k+1}^{(2)}(x) d x= \\
= & \frac{1}{2 h} \int_{x_{k-2}}^{x_{k+2}} f(x+h) S_{k+1}^{(2)}(x+h) d x= \\
= & \frac{1}{2 h} \int_{x_{k-2}}^{x_{k+2}} f(x+h) S_{k}^{(2)}(x) d x,
\end{aligned}
$$

$$
\text { 2) } \begin{aligned}
& F_{k-1}^{(2)}=\frac{1}{2 h} \int_{x_{k-3}}^{x_{k+1}} f(x) S_{k-1}^{(2)}(x) d x= \\
= & \frac{1}{2 h} \int_{x_{k-2}}^{x_{k+2}} f(x-h) S_{k-1}^{(2)}(x-h) d x= \\
= & \frac{1}{2 h} \int_{x_{k-2}}^{x_{k+2}} f(x-h) S_{k}^{(2)}(x) d x .
\end{aligned}
$$

We rewrite formula for $\left(f_{F, n}^{(2)}\right)^{\prime}\left(x_{k}\right)$ in the form

$$
\left(f_{F, n}^{(2)}\right)^{\prime}\left(x_{k}\right)=\frac{1}{2 h} \int_{x_{k-2}}^{x_{k+2}} \phi(x) S_{k}^{(2)}(x) d x,
$$

where

$$
\phi(x)=\frac{f(x+h)-f(x-h)}{2 h},
$$

and by using the first mean value theorem we obtain that there exists a point $\xi_{k} \in\left[x_{k-2}, x_{k+2}\right]$ such that

$$
\left(f_{F, n}^{(2)}\right)^{\prime}\left(x_{k}\right)=\phi\left(\xi_{k}\right) \frac{1}{2 h} \int_{x_{k-2}}^{x_{k+2}} S_{k}^{(2)}(x) d x=\phi\left(\xi_{k}\right) .
$$

Taking into account that according to Lagrange's theorem there exists a point $\mu_{k} \in\left[x_{k-3}, x_{k+3}\right]$ such that

$$
\phi\left(\xi_{k}\right)=\frac{f\left(\xi_{k}+h\right)-f\left(\xi_{k}-h\right)}{2 h}=f^{\prime}\left(\mu_{k}\right),
$$

we obtain that

$$
\left(f_{F, n}^{(2)}\right)^{\prime}\left(x_{k}\right)=f^{\prime}\left(\mu_{k}\right), \text { where } \mu_{k} \in\left[x_{k-3}, x_{k+3}\right] .
$$

From the fact that function $\left(f_{F, n}^{(2)}\right)^{\prime}$ on interval $\left[x_{k-1}, x_{k}\right]$ is linear for $x \in\left[x_{k-1}, x_{k}\right]$ we obtain

$$
\left(f_{F, n}^{(2)}\right)^{\prime}(x)=f^{\prime}\left(\mu_{k-1}\right) \frac{x_{k}-x}{h}+f^{\prime}\left(\mu_{k}\right) \frac{x-x_{k-1}}{h} .
$$

Now we are ready to compare values $\left(f_{F, n}^{(2)}\right)^{\prime}(x)$ and $f^{\prime}(x)$ at arbitrary point $x$ from the interval $\left[x_{k-1}, x_{k}\right]$ :

$$
\begin{gathered}
f^{\prime}(x)-\left(f_{F, n}^{(2)}\right)^{\prime}(x)= \\
=f^{\prime}(x)-\left(f^{\prime}\left(\mu_{k-1}\right) \frac{x_{k}-x}{h}+f^{\prime}\left(\mu_{k}\right) \frac{x-x_{k-1}}{h}\right)= \\
=\left(f^{\prime}(x)-f^{\prime}\left(\mu_{k-1}\right)\right) \frac{x_{k}-x}{h}+ \\
+\left(f^{\prime}(x)-f^{\prime}\left(\mu_{k}\right)\right) \frac{x-x_{k-1}}{h} .
\end{gathered}
$$

If $x \in\left[x_{k-1}, x_{k}\right]$ and $\mu_{k-1} \in\left[x_{k-4}, x_{k+2}\right]$, then

$$
\left|f^{\prime}(x)-f^{\prime}\left(\mu_{k-1}\right)\right| \leq \omega\left(4 h, f^{\prime}\right) .
$$

Conversely, if $x \in\left[x_{k-1}, x_{k}\right]$ and $\mu_{k} \in\left[x_{k-3}, x_{k+3}\right]$, then

$$
\left|f^{\prime}(x)-f^{\prime}\left(\mu_{k}\right)\right| \leq \omega\left(4 h, f^{\prime}\right) .
$$

Therefore

$$
\begin{gathered}
\left|f^{\prime}(x)-\left(f_{F, n}^{(2)}\right)^{\prime}(x)\right| \leq \omega\left(4 h, f^{\prime}\right) \frac{x_{k}-x}{h}+ \\
+\omega\left(4 h, f^{\prime}\right) \frac{x-x_{k-1}}{h}=\omega\left(4 h, f^{\prime}\right) .
\end{gathered}
$$


Theorem 11. Let $f$ be a function from $C^{2}\left(\left[a_{3}, b_{3}\right]\right)$ (i.e. twice continuously differentiable on interval $\left.\left[a_{3}, b_{3}\right]\right)$ and $f_{F, n}^{(3)}$ be its inverse $F^{(3)}$-transform with respect to the third degree spline based uniform generalized fuzzy 3 -partition of $[a, b]$, where

$a_{3}=a-5 h, b_{3}=b+5 h, h=(b-a) /(n-1), n \geq 2$.

Then for all $x \in[a, b]$ holds

$$
\left|f^{\prime \prime}(x)-\left(f_{F, n}^{(3)}\right)^{\prime \prime}(x)\right| \leq \omega\left(6 h, f^{\prime \prime}\right),
$$

where $\omega\left(6 h, f^{\prime \prime}\right)$ is the modulus of continuity of $f^{\prime \prime}$ on $\left[a_{3}, b_{3}\right]$ according to $6 h$.

Proof. By differentiating the formula

$$
f_{F, n}^{(3)}(x)=\frac{1}{3} \sum_{k=-1}^{n+2} F_{k}^{(3)} S_{k}^{(3)}(x),
$$

where

$$
F_{k}^{(3)}=\frac{1}{3 h} \int_{x_{k-3}}^{x_{k-3}} f(x) S_{k}^{(3)}(x) d x
$$

and

$$
\int_{x_{k-3}}^{x_{k-3}} S_{k}^{(3)}(x) d x=3 h
$$

we obtain that the derivative $\left(f_{F, n}^{(3)}\right)^{\prime \prime}$ is the first degree spline

$$
\left(f_{F, n}^{(3)}\right)^{\prime \prime}(x)=\frac{1}{3} \sum_{k=-1}^{n+2} F_{i}^{(3)}\left(S_{i}^{(3)}\right)^{\prime \prime}(x) .
$$

with values

$$
\left(f_{F, n}^{(3)}\right)^{\prime \prime}\left(x_{k}\right)=\frac{1}{3} \sum_{i=k-2}^{k+2} F_{i}^{(3)}\left(S_{i}^{(3)}\right)^{\prime \prime}\left(x_{k}\right) .
$$

By inserting the values

$$
\begin{gathered}
\left(S_{k}^{(3)}\right)^{\prime}\left(x_{k}\right)=0,\left(S_{k-2}^{(3)}\right)^{\prime}\left(x_{k}\right)=\left(S_{k+2}^{(3)}\right)^{\prime}\left(x_{k}\right)= \\
=-\left(S_{k-1}^{(3)}\right)^{\prime \prime}\left(x_{k}\right)=-\left(S_{k+1}^{(3)}\right)^{\prime \prime}\left(x_{k}\right)=\frac{1}{h^{2}},
\end{gathered}
$$

we get

$$
\left(f_{F, n}^{(3)}\right)^{\prime \prime}\left(x_{k}\right)=\frac{F_{k-2}^{(3)}-F_{k-1}^{(3)}-F_{k+1}^{(3)}+F_{k+2}^{(3)}}{3 h^{2}}
$$

and express $F_{k-2}^{(3)}, F_{k-1}^{(3)}, F_{k+1}^{(3)}, F_{k+2}^{(3)}$ by using $S_{k}^{(3)}$ :

$$
\begin{aligned}
& \text { 1) } F_{k-2}^{(3)}=\frac{1}{3 h} \int_{x_{k-5}}^{x_{k+1}} f(x) S_{k-2}^{(3)}(x) d x= \\
&=\frac{1}{3 h} \int_{x_{k-3}}^{x_{k+3}} f(x-2 h) S_{k-2}^{(3)}(x-2 h) d x= \\
&=\frac{1}{3 h} \int_{x_{k-3}}^{x_{k+3}} f(x-2 h) S_{k}^{(3)}(x) d x,
\end{aligned}
$$

$$
\text { 2) } \begin{aligned}
& F_{k-1}^{(3)}=\frac{1}{3 h} \int_{x_{k-4}}^{x_{k+2}} f(x) S_{k-1}^{(3)}(x) d x= \\
= & \frac{1}{3 h} \int_{x_{k-3}}^{x_{k+3}} f(x-h) S_{k-1}^{(3)}(x-h) d x= \\
= & \frac{1}{3 h} \int_{x_{k-3}}^{x_{k+3}} f(x-h) S_{k}^{(3)}(x) d x,
\end{aligned}
$$

$$
\begin{aligned}
& \text { 3) } F_{k+1}^{(3)}=\frac{1}{3 h} \int_{x_{k-2}}^{x_{k+4}} f(x) S_{k+1}^{(3)}(x) d x= \\
& =\frac{1}{3 h} \int_{x_{k-3}}^{x_{k+3}} f(x+h) S_{k+1}^{(3)}(x+h) d x= \\
& =\frac{1}{3 h} \int_{x_{k-3}}^{x_{k+3}} f(x+h) S_{k}^{(3)}(x) d x,
\end{aligned}
$$

4) $F_{k+2}^{(3)}=\frac{1}{3 h} \int_{x_{k-1}}^{x_{k+5}} f(x) S_{k+2}^{(3)}(x) d x=$

$$
\begin{gathered}
=\frac{1}{3 h} \int_{x_{k-3}}^{x_{k+3}} f(x+2 h) S_{k+2}^{(3)}(x+2 h) d x= \\
=\frac{1}{3 h} \int_{x_{k-3}}^{x_{k+3}} f(x+2 h) S_{k}^{(3)}(x) d x .
\end{gathered}
$$

We rewrite the formula for $\left(f_{F, n}^{(3)}\right)^{\prime \prime}\left(x_{k}\right)$ in the form

$$
\left(f_{F, n}^{(3)}\right)^{\prime \prime}\left(x_{k}\right)=\frac{1}{3 h} \int_{x_{k-3}}^{x_{k+3}} \phi(x) S_{k}^{(3)}(x) d x,
$$

where

$$
\begin{gathered}
\phi(x)= \\
=\frac{f(x+2 h)-f(x+h)-f(x-h)+f(x-2 h)}{3 h^{2}},
\end{gathered}
$$

and by using the first mean value theorem we obtain that there exists a point $\xi_{k} \in\left[x_{k-3}, x_{k+3}\right]$ such that

$$
\left(f_{F, n}^{(3)}\right)^{\prime \prime}\left(x_{k}\right)=\phi\left(\xi_{k}\right) \frac{1}{3 h} \int_{x_{k-3}}^{x_{k+3}} S_{k}^{(3)}(x) d x=\phi\left(\xi_{k}\right) .
$$

The obtained result is modified by using NewtonLeibniz formula and the first mean value theorem: there exists a point $\zeta_{k} \in\left[\xi_{k}-2 h, \xi_{k}-h\right]$ such that

$$
\begin{gathered}
\phi\left(\xi_{k}\right)=\frac{1}{3 h^{2}}\left(\int_{\xi_{k+h}}^{\xi_{k+2 h}} f^{\prime}(t) d t-\int_{\xi_{k-2 h}}^{\xi_{k-h}} f^{\prime}(t) d t\right)= \\
=\frac{1}{3 h^{2}} \int_{\xi_{k-2 h}}^{\xi_{k-h}}\left(f^{\prime}(t+3 h)-f^{\prime}(t)\right) d t= \\
=\frac{f^{\prime}\left(\zeta_{k}+3 h\right)-f^{\prime}\left(\zeta_{k}\right)}{3 h} .
\end{gathered}
$$

Taking into account that according to Lagrange's theorem there exists a point $\mu_{k} \in\left[\zeta_{k}, \zeta_{k}+3 h\right]$ such that

$$
\phi\left(\xi_{k}\right)=\frac{f^{\prime}\left(\zeta_{k}+3 h\right)-f^{\prime}\left(\zeta_{k}\right)}{3 h}=f^{\prime \prime}\left(\mu_{k}\right),
$$

we underline that if $\xi_{k} \in\left[x_{k-3}, x_{k+3}\right]$, then $\zeta_{k} \in$ $\left[x_{k-5}, x_{k+2}\right]$ and $\mu_{k} \in\left[x_{k-5}, x_{k+5}\right]$, and obtain that

$$
\left(f_{F, n}^{(3)}\right)^{\prime \prime}\left(x_{k}\right)=f^{\prime \prime}\left(\mu_{k}\right)
$$


for some $\mu_{k} \in\left[x_{k-5}, x_{k+5}\right]$.

From the fact that function $\left(f_{F, n}^{(3)}\right)^{\prime \prime}$ on interval $\left[x_{k-1}, x_{k}\right]$ is a linear we obtain for $x \in\left[x_{k-1}, x_{k}\right]$

$$
\begin{gathered}
\left(f_{F, n}^{(3)}\right)^{\prime \prime}(x)= \\
=\left(f_{F, n}^{(3)}\right)^{\prime \prime}\left(x_{k-1}\right) \frac{x_{k}-x}{h}+\left(f_{F, n}^{(3)}\right)^{\prime \prime}\left(x_{k}\right) \frac{x-x_{k-1}}{h}= \\
=f^{\prime \prime}\left(\mu_{k-1}\right) \frac{x_{k}-x}{h}+f^{\prime \prime}\left(\mu_{k}\right) \frac{x-x_{k-1}}{h} .
\end{gathered}
$$

Now we are ready to compare values $\left(f_{F, n}^{(3)}\right)^{\prime \prime}(x)$ and $f^{\prime \prime}(x)$ at arbitrary point $x \in\left[x_{k-1}, x_{k}\right]$ :

$$
\begin{gathered}
f^{\prime \prime}(x)-\left(f_{F, n}^{(3)}\right)^{\prime \prime}(x)= \\
=f^{\prime \prime}(x)-\left(f^{\prime \prime}\left(\mu_{k-1}\right) \frac{x_{k}-x}{h}+f^{\prime \prime}\left(\mu_{k}\right) \frac{x-x_{k-1}}{h}\right)= \\
=\left(f^{\prime \prime}(x)-f^{\prime \prime}\left(\mu_{k-1}\right)\right) \frac{x_{k}-x}{h}+ \\
+\left(f^{\prime \prime}(x)-f^{\prime \prime}\left(\mu_{k}\right)\right) \frac{x-x_{k-1}}{h} .
\end{gathered}
$$

If $x \in\left[x_{k-1}, x_{k}\right]$ and $\mu_{k-1} \in\left[x_{k-5}, x_{k+3}\right]$, then

$$
\left|f^{\prime \prime}(x)-f^{\prime \prime}\left(\mu_{k-1}\right)\right| \leq \omega\left(6 h, f^{\prime \prime}\right) .
$$

Conversely, if $x \in\left[x_{k-1}, x_{k}\right], \mu_{k} \in\left[x_{k-4}, x_{k+4}\right]$, then

$$
\left|f^{\prime \prime}(x)-f^{\prime \prime}\left(\mu_{k}\right)\right| \leq \omega\left(6 h, f^{\prime \prime}\right) .
$$

Now we have

$$
\begin{aligned}
& \left|f^{\prime \prime}(x)-\left(f_{F, n}^{(3)}\right)^{\prime \prime}(x)\right| \leq \omega\left(6 h, f^{\prime \prime}\right) \frac{x_{k}-x}{h}+ \\
& \quad+\omega\left(6 h, f^{\prime \prime}\right) \frac{x-x_{k-1}}{h}=\omega\left(6 h, f^{\prime \prime}\right) .
\end{aligned}
$$

\section{Conclusions}

In this paper a new type of fuzzy partitions based on polynomial splines are considered. The main attention is paid to approximation properties of $F^{(m)}$ transforms defined with respect to spline based generalized fuzzy $m$-partition. We prove the error bounds for approximation of an original function and its derivatives in the context of the classical approximation theory.

The obtained results allow us to propose the hypothesis that for a given function $f$ from $C^{m-1}\left(\left[a_{m}, b_{m}\right]\right), m \geq 1$, its inverse $F^{(m)}$-transform $f_{F, n}^{(m)}, n \geq 2$, with respect to spline based uniform generalized fuzzy $m$-partition

$$
S_{k}^{(m)}, k=-m+2,-m+3, \ldots, n+m-1,
$$

on $[a, b]$, where

$$
\begin{gathered}
a_{m}=a-(2 m-1) h, b_{m}=b+(2 m+1) h, \\
h=(b-a) /(n-1),
\end{gathered}
$$

provides the following result for derivative approximation:

$$
\left|f^{(m-1)}(x)-\left(f_{F, n}^{(3)}\right)^{(m-1)}(x)\right| \leq \omega\left(2 m h, f^{(m-1)}\right)
$$

for all $x \in[a, b]$, where $\omega\left(2 m h, f^{(m-1)}\right)$ is the modulus of continuity of $f^{(m-1)}$ on $\left[a_{m}, b_{m}\right]$ according to $2 m h$.

At the present moment this error bound is proved for $m=1,2,3$. Our future work will be devoted to the proving of the general case.

\section{Acknowledgements}

The authors would like to express their thanks to I. Perfilieva for her attention to this research. This work has been partially supported by the project 2009/0223/1DP/1.1.1.2.0/09/APIA/VIAA/008.

\section{References}

[1] B. Bede and I.J. Rudas, Approximation properties of fuzzy transforms, Fuzzy Sets and Systems, 180:20-40, Elsevier, 2011.

[2] C. de Boor. A practical guide to splines, Springer, 2001.

[3] F. Di Martino, V. Loia, I. Perfilieva and S. Sessa, An image coding/decoding method based on direct and inverse fuzzy transforms, International Journal of Approximate Reasoning, 48(1): 110131, Elsevier, 2008.

[4] F. Di Martino, V. Loia and S. Sessa, A segmentation method for images compressed by fuzzy transforms, Fuzzy Sets and Systems, 161(1): 5674, Elsevier, 2010.

[5] F. Di Martino, V. Loia and S. Sessa, Fuzzy transforms method and attribute dependency in data analysis, Information Sciences, 180(4): 493-505, Elsevier, 2010.

[6] I. Perfilieva, Fuzzy transforms: Theory and applications, Fuzzy Sets and Systems, 157:9931004, Elsevier, 2006.

[7] I. Perfilieva and B. De Baets, Fuzzy transforms of monotone functions with application to image compression, Information Sciences, 180(17): 3304-3315, Elsevier, 2010.

[8] I. Perfilieva, M. Dankova and B. Bede, Towards a higher degree F-transform, Fuzzy Sets and Systems, 180:3-19, Elsevier, 2011.

[9] I. Perfilieva and V. Kreinovich, Fuzzy transform as a new paradigm in fuzzy modeling, Fuzzy Sets and Systems, 180:1-2, Elsevier, 2011.

[10] I. Perfilieva and V. Kreinovich, Fuzzy transforms of higher order approximate derivatives: A theorem, Fuzzy Sets and Systems, 180:55-68, Elsevier, 2011.

[11] I. Perfilieva, V. Novak and A. Dvorak, Fuzzy transform in the analysis of data, International Journal of Approximate Reasoning, 48(1): 3646, Elsevier, 2008.

[12] L. Stefanini, F-transform with parametric generalized fuzzy partitions, Fuzzy Sets and Systems, 180:98-120, Elsevier, 2011.

[13] L.L. Schumaker. Spline functions: basic theory, Cambridge University Press, 2007. 